\title{
The Interactions of $\mathrm{N}$-Soliton Solutions for the Generalized 2 + 1-Dimensional Variable-Coefficient Fifth-Order KdV Equation
}

\author{
Xiangrong Wang, Xiaoen Zhang, Yong Zhang, and Huanhe Dong \\ College of Mathematics and Systems Science, Shandong University of Science and Technology, Qingdao 266590, China \\ Correspondence should be addressed to Xiaoen Zhang; xezhang19890309@163.com
}

Received 16 September 2015; Accepted 12 November 2015

Academic Editor: Giorgio Kaniadakis

Copyright (C) 2015 Xiangrong Wang et al. This is an open access article distributed under the Creative Commons Attribution License, which permits unrestricted use, distribution, and reproduction in any medium, provided the original work is properly cited.

\begin{abstract}
A generalized $(2+1)$-dimensional variable-coefficient $\mathrm{KdV}$ equation is introduced, which can describe the interaction between a water wave and gravity-capillary waves better than the $(1+1)$-dimensional $\mathrm{KdV}$ equation. The $\mathrm{N}$-soliton solutions of the $(2+1)$ dimensional variable-coefficient fifth-order KdV equation are obtained via the Bell-polynomial method. Then the soliton fusion, fission, and the pursuing collision are analyzed depending on the influence of the coefficient $e^{A_{i j}}$; when $e^{A_{i j}}=0$, the soliton fusion and fission will happen; when $e^{A_{i j}} \neq 0$, the pursuing collision will occur. Moreover, the Bäcklund transformation of the equation is gotten according to the binary Bell-polynomial and the period wave solutions are given by applying the Riemann theta function method.
\end{abstract}

\section{Introduction}

In soliton theory, the nonlinear evolution equations (NLEEs) [1-3] have described natural phenomena in many aspects, such as in the nonlinear phonology [4, 5], water waves [6], hydromechanics [7], and super symmetry. Especially in the hydromechanics, the nonlinear evolution equations can explain the interaction of the waves by the different dispersion relations. As for the NLEEs, there are many methods to get the solutions, like the Hirota method [8-13], the Darboux transformation, Bell-polynomial approach [14-20], Bäcklund transformations [21, 22], and so on [23]. One can get the $N$ soliton solutions and analyze the interactions of the waves based on the Bell-polynomial approach.

Soliton interaction can be split into elastic and inelastic. As for the elastic, the amplitudes, velocities, and shapes of the soliton can be brought into correspondence with the initial soliton, but for the inelastic collision, after the interaction, one soliton can be divided into two or more solitons, a phenomenon called soliton fission, or contrarily, two or more solitons can be merged into one soliton which is called soliton fusion. What is more, the variable coefficient of the equation can lead to the soliton fission and fusion. In [14], the variablecoefficient KdV equation can be applied to describe the largeamplitudes internal waves of the atmosphere and the ocean. In recent years, the general $\mathrm{KdV}$ equation had been expanded to the fifth-order $\mathrm{KdV}$ equation and the generalized $(2+$ 1)-dimensional Korteweg-de Vries equation whose bilinear Bäcklund transformation and Darboux covariant Lax pair have been obtained.

In this paper, we introduced a generalized variablecoefficient fifth-order KdV equation as follows:

$$
\begin{aligned}
u_{t}+a(t) u u_{x}+b(t) u_{x x x}+c(t) u^{2} u_{x}+d(t) u_{x} u_{2 x} \\
+e(t) u u_{x x x}+f(t) u_{x x x x x}+g(t) u_{x} \partial_{x}^{-1} u_{y} \\
+h(t) u_{x x y}+k(t) u u_{y}=0,
\end{aligned}
$$

where $u$ is a real function of space $x, y$, and time $t$ and $a(t), b(t), c(t), d(t), e(t), f(t), g(t), h(t)$, and $k(t)$ are the functions of $t$. It can be used to describe the gravity-capillary wave on a fluid interface, which is influenced by both the effects of surface tension and gravity as well as by fluid inertia. Equation (1) has special issues. 
(1) When $g(t)=h(t)=k(t)=0, a(t)=6, b(t)=1$, and $c(t)=d(t)=e(t)=f(t)=$ constant, it can be changed into the fifth-order KdV equation [24],

$$
\begin{gathered}
u_{t}+6 u u_{x}+u_{x x x}+c u^{2} u_{x}+d u_{x} u_{2 x}+e u u_{x x x} \\
+f u_{x x x x x}=0,
\end{gathered}
$$

whose Darboux transformation, bilinear representation, $\mathrm{N}$ soliton solutions, and bilinear Bäcklund transformation have been obtained.

(2) When $a(t)=6 b(t), k(t)=4 h(t)=2 g(t), c(t)=d(t)=$ $e(t)=f(t)=0$, and $b(t)=h(t)=$ constants, (1) can be changed into generalized $(2+1)$-dimensional Korteweg-de Vries equation [25],

$$
\begin{aligned}
u_{t} & +6 b u u_{x}+b u_{x x x}+2 h u_{x} \partial_{x}^{-1} u_{y}+h u_{x x y}+4 h u u_{y} \\
& =0
\end{aligned}
$$

whose Bell-polynomial, Bäcklund transformation, and Darboux covariant Lax pair have been got.

(3) When $a(t)=3, b(t)=\gamma, d(t)=2, e(t)=1, f(t)=$ $2 / 15$, and $c(t)=g(t)=h(t)=k(t)=0$, (1) takes the form of

$$
u_{t}+3 u u_{x}-\gamma u_{x x x}+2 u_{x} u_{2 x}+u u_{x x x}+\frac{2}{15} u_{x x x x x}=0
$$

which occurs to a weakly nonlinear long-wave approximation to the general gravity-capillary water-wave problem and $\gamma$ is a real scaled parameter.

The focus of the paper is to get the $N$-soliton solutions of the generalized variable-coefficient fifth-order $(2+1)$ dimensional equation and analyze the interaction of the water wave and the gravity-capillary wave [26-30]. The details of the paper are as follows: Section 2 introduces a variablecoefficient fifth-order $(2+1)$-dimensional $\mathrm{KdV}$ equation. The approach and the properties of the Bell-polynomial are presented in Section 3 and then give rise to the $N$-soliton solutions of the equation based on the Hirota approach. In the final part, we explain the soliton fission and fusion and the soliton pursuing collision of the variable-coefficient fifth-order $(2+1)$-dimensional $\mathrm{KdV}$ equation according to the different coefficients $e^{A_{i j}}$. Furthermore, the Bäcklund transformation is given.

\section{The Introduction of the Bell-Polynomial}

To start with, we briefly introduce the basic concepts and the properties of the Bell-polynomial.

(1) Let $\theta$ be a function of the variable $x$; then the formula [25]

$$
Y_{n x}=Y_{n}\left(\theta_{x}, \theta_{x x}, \ldots, \theta_{n x}\right)=e^{-\theta} \partial_{x}^{n} e^{\theta}, \quad(n=1,2, \ldots)
$$

is a polynomial concerning $\theta$ with respect to $x$, which is the definition of the one-dimensional Bell-polynomial.
Moreover, we can calculate the initial explicit expressions by the definition as follows:

$$
\begin{aligned}
& Y_{x}=\theta_{x}, \\
& Y_{2 x}=\theta_{2 x}+\theta_{x}^{2}, \\
& Y_{3 x}=\theta_{x x x}+3 \theta_{x} \theta_{x x}+\theta_{x}^{2}, \\
& Y_{4 x}=\theta_{4 x}+4 \theta_{x} \theta_{3 x}+3 \theta_{x x}^{2}+6 \theta_{x}^{2} \theta_{x x}+\theta_{x}^{4} .
\end{aligned}
$$

(2) Take $\theta$ as a $C^{\infty}$ multivariables function; then the definition of the multivariables Bell-polynomial is as follows:

$$
\begin{aligned}
& Y_{n_{1} x_{1} n_{2} x_{2} \cdots n_{r} x_{r}}(\theta) \\
& \quad \equiv Y_{n_{1}, n_{2}, \ldots, n_{r}}\left(\theta_{l_{1} x_{1}, \ldots, l_{r} x_{r}}\left(1 \leq l_{i} \leq n_{i}, 0 \leq i \leq r\right)\right) \\
& \quad=e^{-\theta} \partial_{x_{1}}^{n_{1}} \partial_{x_{2}}^{n_{2}} \cdots \partial_{x_{r}}^{n_{r}} e^{\theta} .
\end{aligned}
$$

As for a special function $\theta$ with the variables $x, y$, we give rise to the following several initial values under the definition of the multivariables Bell-polynomial:

$$
\begin{aligned}
Y_{x, y}(\theta) & =\theta_{x, y}+\theta_{x} \theta_{y} \\
Y_{x, 2 y}(\theta) & =\theta_{x, 2 y}+\theta_{x} \theta_{2 y}+2 \theta_{x, y} \theta_{y}+\theta_{x} \theta_{y}^{2}+\cdots .
\end{aligned}
$$

In view of the multivariables Bell-polynomial, the multivariables binary Bell-polynomial can be defined as follows:

$$
\begin{aligned}
& \mathscr{Y}_{n_{1} x_{1}, \ldots, n_{r} x_{r}}(\varphi, \psi) \\
& \left.\equiv Y_{n_{1}, \ldots, n_{r}}(\theta)\right|_{\theta_{l_{1} x_{1}, \ldots, l_{r} x_{r}}}= \begin{cases}\varphi_{l_{1} x_{1}, \ldots, l_{r} x_{r}} & \sum_{i=1}^{r} l_{i} \text { is odd } \\
\psi_{l_{1} x_{1}, \ldots, l_{r} x_{r}} & \Sigma_{i=1}^{r} l_{i} \text { is even, }\end{cases}
\end{aligned}
$$

where $\varphi$ and $\psi$ both are the $C^{\infty}$ function with the variables $x_{1}, x_{2}, \ldots, x_{r}$; likewise, we can take some of the expressions depending on (9); for example,

$$
\begin{aligned}
\mathscr{Y}_{x}(\varphi) & =\varphi_{x}, \\
\mathscr{Y}_{2 x}(\varphi, \psi) & =\varphi_{x}^{2}+\psi_{x x}, \\
\mathscr{Y}_{x, y} & =\psi_{x, y}+\varphi_{x} \varphi_{y}, \\
\mathscr{Y}_{3 x}(\varphi, \psi) & =\varphi_{3 x}+3 \varphi_{x} \psi_{2 x}+\varphi_{x}^{3}, \\
\mathscr{Y}_{2 x, y}(\varphi, \psi) & =\varphi_{2 x, y}+2 \varphi_{x} \psi_{x, y}+\varphi_{x}^{2} \varphi_{y}+\varphi_{y} \psi_{2 x} \\
& +\cdots .
\end{aligned}
$$

Next, we study the proposition of the Bell-polynomial.

Proposition 1. Bell-polynomial (9) can be written as the Hirota D-operator through a transformational identity:

$$
\begin{gathered}
\mathscr{Y}_{n_{1} x_{1}, \ldots, n_{r} x_{r}}\left(\varphi=\ln \frac{F}{G}, \psi=\ln F G\right) \\
=(F \cdot G)^{-1} D_{x_{1}}^{n_{1}} \cdots D_{x_{r}}^{n_{r}} F \cdot G,
\end{gathered}
$$


where the Hirota operator is defined by

$$
\begin{gathered}
D_{x_{1}}^{n_{1}} \cdots D_{x_{r}}^{n_{r}} F \cdot G=\left(\partial_{x_{1}}-\partial_{x_{1}^{\prime}}\right)^{n_{1}} \cdots\left(\partial_{x_{r}}-x_{r}^{\prime}\right) \\
\left.\cdot F\left(x_{1}, \ldots, x_{r}\right) G\left(x_{1}^{\prime}, \ldots, x_{r}^{\prime}\right)\right|_{x_{1}^{\prime}=x_{1}, \ldots, x_{r}^{\prime}=x_{r}} .
\end{gathered}
$$

Especially when $F=G$, (11) can be read as

$$
\begin{aligned}
& F^{-2} D_{x_{1}}^{n_{1}} \cdots D_{x_{r}}^{n_{r}} F^{2}=\mathscr{Y}_{n_{1} x_{1}, \ldots, n_{r} x_{r}}(\varphi=0, \psi=2 \ln F) \\
& \quad= \begin{cases}0, & \sum_{i=1}^{r} n_{i} \text { is odd }, \\
\mathscr{P}_{n_{1} x_{1}, \ldots, n_{r} x_{r}}(q), & \sum_{i=1}^{r} n_{i} \text { is even. }\end{cases}
\end{aligned}
$$

In (13), the Bell-polynomial is significant if and only if when $\sum_{i=1}^{r} n_{i}$ is even, one redefines a P-polynomial:

$$
\mathscr{P}_{n_{1} x_{1}, \ldots, n_{r} x_{r}}(q)=\mathscr{Y}_{n_{1} x_{1}, \ldots, n_{r} x_{r}}(\varphi=0, \psi=2 \ln F),
$$

when $n_{1}+n_{2}+\ldots+n_{r}$ is even. The initial few P-polynomials are

$$
\begin{aligned}
\mathscr{P}_{2 x}(q) & =q_{2 x}, \\
\mathscr{P}_{4 x}(q) & =q_{4 x}+3 q_{2 x}^{2}, \\
\mathscr{P}_{2 x, 2 y}(q) & =q_{2 x, 2 y}+q_{2 x} q_{2 y}+2 q_{x, y}^{2}, \\
\mathscr{P}_{x, y}(q) & =q_{x, y}, \\
\mathscr{P}_{3 x, y}(q) & =q_{3 x, y}+3 q_{x, y} q_{2 x}, \\
\mathscr{P}_{6 x}(q) & =q_{6 x}+15 q_{2 x} q_{4 x}+15 q_{2 x}^{3}+\cdots .
\end{aligned}
$$

As for the NLEEs, (9) and (15) are important to get the Nsoliton solutions; one can get the bilinear equation provided that the NLEEs can express the linear combination of the Ppolynomials.

\section{The $N$-Soliton Solutions of the Variable- Coefficient Fifth-Order KdV Equation}

As for (1), if we take

$$
\begin{aligned}
& a(t)=6 b(t), \\
& d(t)=\frac{2}{3} c(t), \\
& e(t)=\frac{1}{3} c(t), \\
& f(t)=\frac{1}{30} c(t), \\
& g(t)=2 h(t), \\
& k(t)=4 h(t),
\end{aligned}
$$

then (1) can be rewritten as

$$
\begin{aligned}
u_{t} & +b(t)\left(6 u u_{x}+u_{x x x}\right) \\
& +\frac{1}{30} c(t)\left(30 u^{2} u_{x}+20 u_{x} u_{2 x}+10 u u_{x x x}+u_{x x x x x}\right) \\
& +h(t)\left(2 u_{x} \partial_{x}^{-1} u_{y}+u_{x x y}+4 u u_{y}\right)=0 .
\end{aligned}
$$

By virtue of the transformation $u=Q_{x x},(17)$ can be changed into

$$
\begin{aligned}
& Q_{x x t}+b(t)\left(6 Q_{x x} Q_{x x x}+Q_{5 x}\right)+\frac{1}{30} c(t) \\
& \cdot\left(30 Q_{x x}^{2} Q_{x x x}+20 Q_{x x x} Q_{4 x}+10 Q_{x x} Q_{5 x}+Q_{7 x}\right) \\
& +h(t)\left(2 Q_{3 x} Q_{x, y}+Q_{4 x, y}+4 Q_{x x} Q_{x x y}\right)=0 .
\end{aligned}
$$

What is more, we can obtain the following formula by making use of the integral to (18) with respect to the variable $x$ :

$$
\begin{aligned}
Q_{x t}+ & b(t)\left(3 Q_{x x}^{2}+Q_{4 x}\right) \\
& +\frac{1}{30} c(t)\left(10 Q_{x x}^{3}+10 Q_{x x} Q_{4 x}+5 Q_{3 x}^{2}+Q_{6 x}\right) \\
& +h(t)\left(2 Q_{2 x} Q_{x, y}+Q_{3 x, y}+2 \partial_{x}^{-1} Q_{x x} Q_{x x y}\right)=0 .
\end{aligned}
$$

Expression (19) is changed as follows with the aid of $P$ polynomial (15):

$$
\begin{aligned}
\mathscr{P}_{x, t}(Q)+b(t) \mathscr{P}_{4 x}(Q)+\frac{c(t)}{30} \mathscr{P}_{6 x}(Q) \\
-\frac{c(t)}{18}\left(\partial_{x}^{2} \mathscr{P}_{4 x}(Q)+3 Q_{2 x} \mathscr{P}_{4 x}(Q)\right. \\
\left.-\partial_{x}^{-1}\left(\partial_{x}^{3} \mathscr{P}_{4 x}(Q)+6 Q_{2 x} \partial_{x} \mathscr{P}_{4 x}(Q)\right)\right)+\frac{2}{3} h(t) \\
\cdot\left(\mathscr{P}_{3 x, y}(Q)\right)+\frac{1}{3} h(t) \partial_{x}^{-1} \partial_{y}\left(\mathscr{P}_{4 x}(Q)\right)=0,
\end{aligned}
$$

which cannot be written as the linear combination of $P$ polynomials, so we construct an auxiliary variable $z$ which satisfies

$$
Q_{4 x}+3 Q_{2 x}^{2}+Q_{x, z}=0 .
$$

From (21), we can get a pair of $P$-polynomials as follows:

$$
\begin{aligned}
& \mathscr{P}_{4 x}(Q)+\mathscr{P}_{x, z}(Q)=0, \\
& \mathscr{P}_{x, t}(Q)+b(t) \mathscr{P}_{4 x}(Q)+\frac{c(t)}{30} \mathscr{P}_{6 x}(Q)
\end{aligned}
$$




$$
\begin{aligned}
& -\frac{c(t)}{18}\left(\mathscr{P}_{3 x, z}(Q)+\mathscr{P}_{2 z}(Q)\right)+\frac{2}{3} h(t) \mathscr{P}_{3 x, y}(Q) \\
& -\frac{1}{3} h(t) \mathscr{P}_{y, z}(Q)=0 .
\end{aligned}
$$

Finally, generalized variable-coefficient $(2+1)$-dimensional fifth-order KdV equation (22) can be cast into a bilinear representation based on the transportation $Q=2 \ln f$,

$$
\begin{aligned}
& \left(D_{x}^{4}+D_{x} D_{z}\right) f \cdot f=0 \\
& \left(D_{x} D_{t}+b(t) D_{x}^{4}+\frac{c(t)}{30} D_{x}^{6}-\frac{c(t)}{18}\left(D_{x}^{3} D_{z}+D_{z}^{2}\right)\right. \\
& \left.\quad+\frac{2}{3} h(t) D_{x}^{3} D_{y}-\frac{1}{3} h(t) D_{y} D_{z}\right) f \cdot f=0
\end{aligned}
$$

$$
\begin{aligned}
\gamma_{j} & =k_{j}\left(x+p_{j} y\right)-\int\left(\frac{c(t)}{30} k_{j}^{5}+b(t) k_{j}^{3}+h(t) k_{j}^{3} p_{j}\right) d t \\
e^{A_{i j}} & =\frac{(c(t) / 6)\left(k_{i}-k_{j}\right)^{2}\left(k_{i}^{2}+k_{j}^{2}-k_{i} k_{j}\right)+3 b(t)\left(k_{i}-k_{j}\right)^{2}+h(t)\left(k_{i}-k_{j}\right)\left(p_{i}\left(2 k_{i}-k_{j}\right)-p_{j}\left(2 k_{j}-k_{i}\right)\right)}{(c(t) / 6)\left(k_{i}+k_{j}\right)^{2}\left(k_{i}^{2}+k_{j}^{2}+k_{i} k_{j}\right)+3 b(t)\left(k_{i}+k_{j}\right)^{2}+h(t)\left(k_{i}+k_{j}\right)\left(p_{i}\left(2 k_{i}-k_{j}\right)+p_{j}\left(2 k_{j}-k_{i}\right)\right)},
\end{aligned}
$$

Once the bilinear representation of (19) is given, we can present the $N$-soliton solutions of (23) with the help of Hirota's bilinear approach and the symbolic computation.

After that, we begin to solve (17) on account of the Hirota method; set

$$
f=1+\varepsilon f_{1}+\varepsilon^{2} f_{2}+\varepsilon^{3} f_{3}+\cdots .
$$

Substitute (24) into (23) and compare the powers of $\varepsilon$; then the $N$-soliton solutions of (19) are gotten by making $\varepsilon=1$ as follows:

$$
f=\sum_{\mu_{i}, \mu_{j}=0,1} \exp \left\{\sum_{i>j}^{N} A_{i j} \mu_{i} \mu_{j}+\sum_{j=1}^{N} \mu_{j} \gamma_{j}\right\},
$$

with where $k_{j}$ and $p_{j}$ are both the constants and $\sum_{\mu_{i}, \mu_{j}=0,1}$ indicate summation over all the different possible cases $\mu_{i}, \mu_{j}=$ $0,1(i, j=1,2,3, \ldots)$.

For $N=1$, we can read the one-soliton solution as

$$
u=\frac{1}{2} k_{1}^{2} \operatorname{sech}^{2}\left(\frac{\gamma_{1}}{2}\right)
$$

For $N=2$, the two-soliton solutions can be written as

$$
\begin{aligned}
u= & 2 \frac{k_{1}^{2} e^{\gamma_{1}}+k_{2}^{2} e^{\gamma_{2}}+e^{A_{21}}\left(k_{1}+k_{2}\right)^{2} e^{\gamma_{1}+\gamma_{2}}}{1+e^{\gamma_{1}}+e^{\gamma_{2}}+e^{A_{21}} e^{\gamma_{1}+\gamma_{2}}} \\
& -2 \frac{\left(k_{1} e^{\gamma_{1}}+k_{2} e^{\gamma_{2}}+e^{A_{21}}\left(k_{1}+k_{2}\right) e^{\gamma_{1}+\gamma_{2}}\right)^{2}}{\left(1+e^{\gamma_{1}}+e^{\gamma_{2}}+e^{A_{21}} e^{\gamma_{1}+\gamma_{2}}\right)^{2}} .
\end{aligned}
$$

For $N=3$, we can obtain the three-soliton solutions as

$$
\begin{aligned}
u= & 2\left[\operatorname { l n } \left(1+e^{\gamma_{1}}+e^{\gamma_{2}}+e^{\gamma_{3}}+e^{\gamma_{1}+\gamma_{2}+A_{21}}+e^{\gamma_{1}+\gamma_{3}+A_{31}}\right.\right. \\
& \left.\left.+e^{\gamma_{2}+\gamma_{3}+A_{32}}+e^{\gamma_{1}+\gamma_{2}+\gamma_{3}+A_{21}+A_{31}+A_{32}}\right)\right]_{x x} .
\end{aligned}
$$

\section{The Bäcklund Transformation of the Variable-Coefficient Fifth-Order KdV Equation}

For the NLEEs, the BT method provides a new idea to construct the solutions by the Bell-polynomial. In this section, we will obtain the BT of the known $(2+1)$-dimensional variable-coefficient fifth-order $\mathrm{KdV}$ equation. Suppose $Q, \bar{Q}$ are the two different solutions of (19), and consider the following form:

$$
\begin{aligned}
L= & \frac{1}{30} c(t)\left(10 Q_{x x}^{3} 10 Q_{x x} Q_{4 x}+5 Q_{3 x}^{2}+Q_{6 x}-10 \bar{Q}_{x x}^{3}\right. \\
& \left.-10 \bar{Q}_{x x} \bar{Q}_{4 x}-5 \bar{Q}_{3 x}^{2}+\bar{Q}_{6 x}\right)+h(t)\left(2 Q_{2 x} Q_{x, y}\right. \\
& +Q_{3 x, y}+2 \partial_{x}^{-1} Q_{x x} Q_{x x y}-2 \bar{Q}_{2 x} \bar{Q}_{x, y}-\bar{Q}_{3 x, y} \\
& \left.-2 \partial_{x}^{-1} \bar{Q}_{x x} \bar{Q}_{x x y}\right)+Q_{x t}-\bar{Q}_{x t}+b(t)\left(3 Q_{x x}^{2}+Q_{4 x}\right. \\
& \left.-3 \bar{Q}_{x x}^{2}-\bar{Q}_{4 x}\right)=0
\end{aligned}
$$

In order to obtain the BT of (30), we introduce the mixing variables as

$$
\begin{aligned}
& \varphi=\ln (F \cdot G), \\
& \psi=\ln \left(\frac{F}{G}\right), \\
& Q=\varphi+\psi=2 \ln F, \\
& \bar{Q}=\psi-\varphi=2 \ln G ;
\end{aligned}
$$


then

$$
\begin{aligned}
L= & 2\left(\varphi_{x t}+b(t)\left(6 \varphi_{x x} \psi_{x x}+\varphi_{4 x}\right)+\frac{c(t)}{30}\left(10 \varphi_{2 x}^{3}\right.\right. \\
& +30 \varphi_{2 x} \psi_{2 x}^{2}+10 \varphi_{2 x} \psi_{4 x}+10 \varphi_{4 x} \psi_{2 x}+10 \varphi_{3 x} \psi_{3 x} \\
& \left.+\varphi_{6 x}\right)+\frac{2}{3} h(t)\left(\varphi_{3 x, y}+3 \varphi_{2 x} \psi_{x, y}+3 \varphi_{x, y} \psi_{2 x}\right)+\frac{1}{3} \\
& \left.\cdot h(t) \partial_{x}^{-1} \partial_{y}\left(6 \varphi_{x x} \psi_{x x}+\varphi_{4 x}\right)\right) \\
= & 2 \partial_{x}\left(\mathscr{Y}_{t}(\varphi)+b(t) \mathscr{Y}_{3 x}(\varphi, \psi)+\frac{c(t)}{30} \mathscr{Y}_{5 x}(\varphi, \psi)\right. \\
& \left.+h(t) \mathscr{Y}_{2 x, y}(\varphi, \psi)\right)+R(\varphi, \psi),
\end{aligned}
$$

where

$$
\begin{aligned}
& R(\varphi, \psi)=2\left(b(t)\left(3 \varphi_{2 x} \psi_{3 x}-3 \varphi_{x}^{2} \varphi_{x x}-3 \varphi_{x} \psi_{4 x}\right)\right. \\
& +h(t)\left(2 \varphi_{x, y} \psi_{x x}-2 \varphi_{x} \psi_{x x y}-\partial_{x}\left(\varphi_{x}^{2} \varphi_{y}+\varphi_{y} \psi_{2 x}\right)\right) \\
& +\frac{c(t)}{30}\left(5 \varphi_{2 x} \psi_{4}+15 \varphi_{2 x} \psi_{2 x}^{2}+10 \varphi_{2 x}^{3}-5 \varphi_{x}^{4} \varphi_{2 x}\right. \\
& -30 \varphi_{x}^{2} \varphi_{x x} \psi_{x x}-10 \varphi_{x}^{3} \psi_{x x x}-30 \varphi_{x} \psi_{2 x} \psi_{3 x} \\
& \left.-20 \varphi_{x} \varphi_{x x} \varphi_{3 x}-5 \varphi_{x} \psi_{5 x}-10 \varphi_{x}^{2} \varphi_{4 x}\right)+\frac{1}{3} h(t) \\
& \left.. \partial_{x}^{-1}\left(6 \varphi_{x x y} \psi_{x x}+6 \varphi_{x x} \psi_{2 x, y}\right)\right)
\end{aligned}
$$

Finally, we derive the BT by introducing a spectrum parameter equation as

$$
\mathscr{Y}_{2 x}(\varphi, \psi)=\lambda
$$

and have

$$
\begin{aligned}
L= & 2 \partial_{x}\left(\mathscr{Y}_{t}(\varphi)+b(t) \mathscr{Y}_{3 x}(\varphi, \psi)+\frac{c(t)}{30} \mathscr{Y}_{5 x}(\varphi, \psi)\right. \\
& +h(t) \mathscr{Y}_{2 x, y}(\varphi, \psi)+\left(3 \lambda b(t)+\frac{c(t)}{2} \lambda^{2}\right) \mathscr{Y}_{x}(\varphi) \\
& \left.+3 \lambda h(t) \mathscr{Y}_{y}(\varphi)\right)
\end{aligned}
$$

$$
\begin{aligned}
H f \cdot f & =H\left(D_{x}, D_{y}, D_{t}\right) \sum_{n=-\infty}^{n=\infty} e^{2 \pi i n \gamma+\pi i n^{2} \tau} \sum_{m=-\infty}^{m=\infty} e^{2 \pi i m \gamma+\pi i m^{2} \tau}=\sum_{n=-\infty}^{n=\infty} \sum_{m=-\infty}^{m=\infty} H\left(D_{x}, D_{y}, D_{t}\right) e^{2 \pi i n \gamma+\pi i n^{2} \tau} \cdot e^{2 \pi i m \gamma+\pi i m^{2} \tau} \\
& =\sum_{n=-\infty}^{n=\infty} \sum_{m=-\infty}^{m=\infty} H(2 \pi i(n-m) k, 2 \pi i(n-m) k p, 2 \pi i(n-m) w) e^{2 \pi i(n+m) \gamma+\pi i\left(n^{2}+m^{2}\right) \tau} \\
& =\sum_{q=-\infty}^{q=\infty}\left\{\sum_{n=-\infty}^{n=\infty} H(2 \pi i(2 n-q) k, 2 \pi i(2 n-q) k p, 2 \pi i(2 n-q) w) e^{\pi i\left(n^{2}+(q-n)^{2}\right) \tau}\right\} e^{2 \pi i q \gamma}=\sum_{q=-\infty}^{q=\infty} \bar{H}(q) e^{2 \pi i q \gamma} .
\end{aligned}
$$




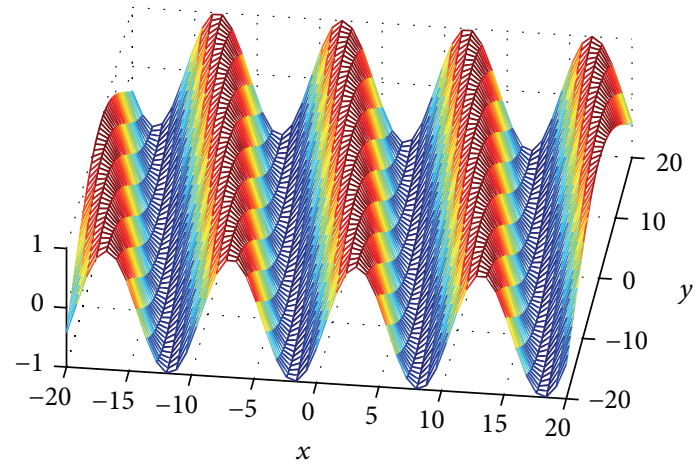

Figure 1: One period wave solution to (17) with the parameters $k=0.1, p=1, t=0.5, \tau=2 i, c(0.5)=30, b(0.5)=1$, and $h(0.5)=1$.

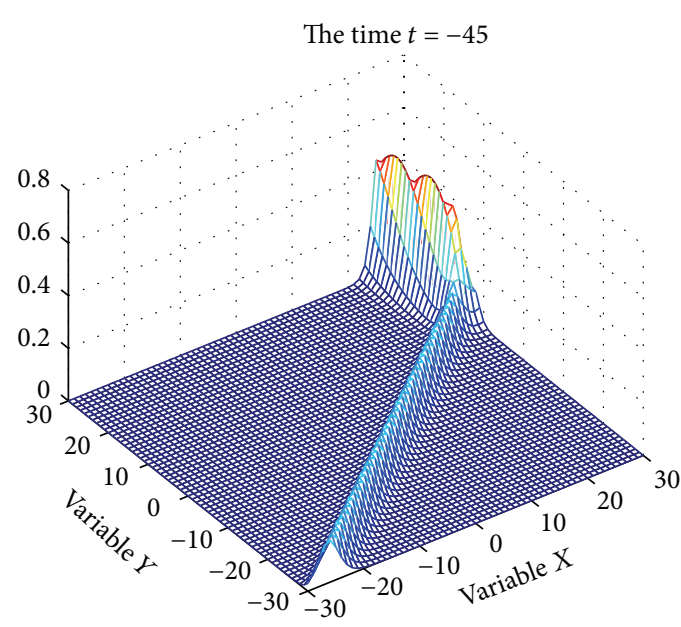

(a)

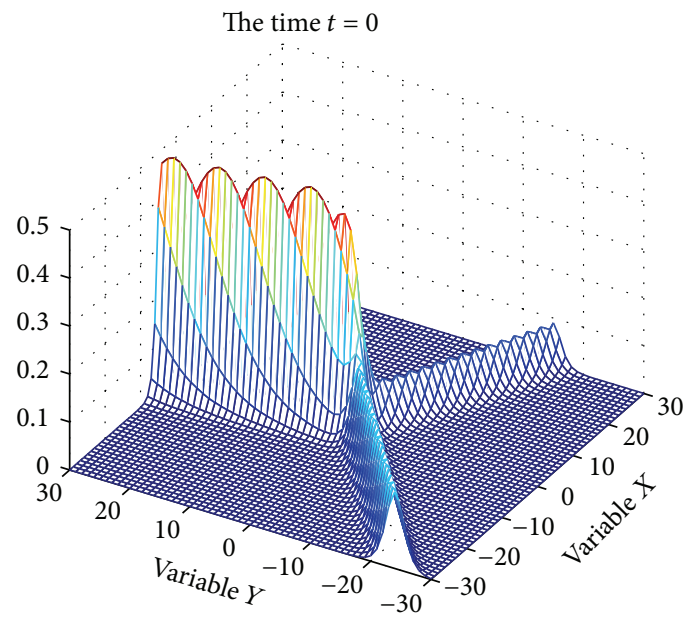

(b)

The time $t=70$

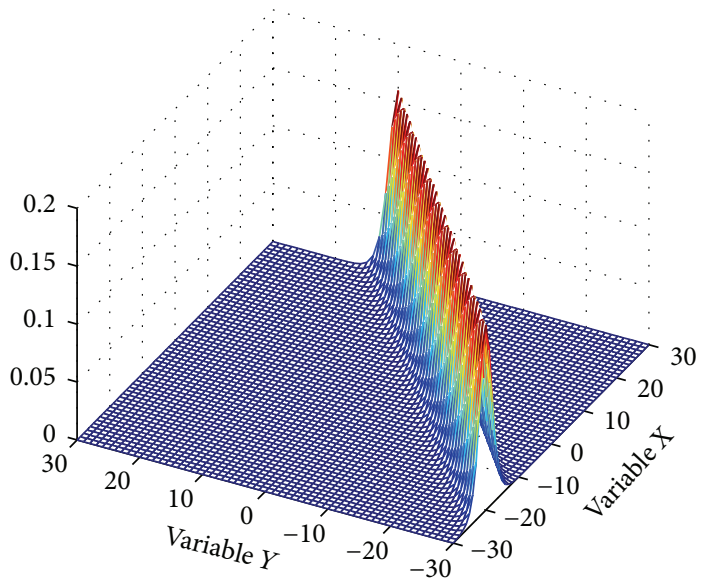

(c)

Figure 2: The process of the soliton fusion for the two solitary waves (46) with $k_{1}=-0.8, k_{2}=0.6, p_{1}=-1.25$, and $p_{2}=2$, and (a) implies $t=-45$, (b) implies $t=0$, and (c) implies $t=70$.

Based on the calculation of (39), we can obtain

$$
\begin{gathered}
\bar{H}(q)=\sum_{n=-\infty}^{n=\infty} H(2 \pi i(2 n-q) k, 2 \pi i(2 n-q) \\
\cdot k p, 2 \pi i(2 n-q) w) e^{\pi i\left(n^{2}+(q-n)^{2}\right) \tau}
\end{gathered}
$$

$$
\begin{aligned}
& =\sum_{h=-\infty}^{h=\infty} H(2 \pi i B k, 2 \pi i B k p, 2 \pi i B w) e^{\pi i\left(n^{2}+(q-h-2)^{2}\right) \tau} \\
& \text { - } e^{2 \pi i(q-1) \tau} \text {, }
\end{aligned}
$$




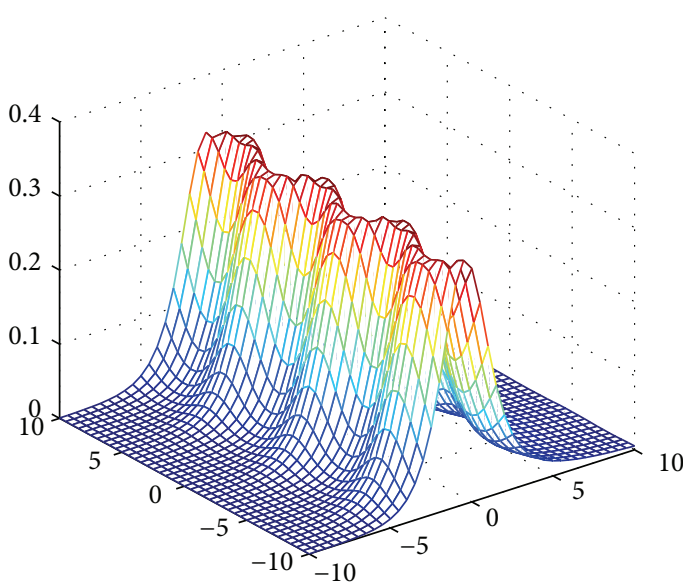

(a)

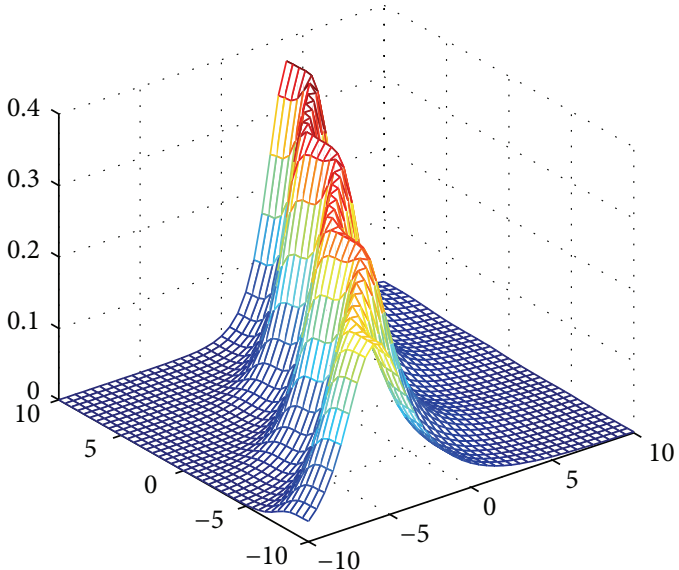

(b)

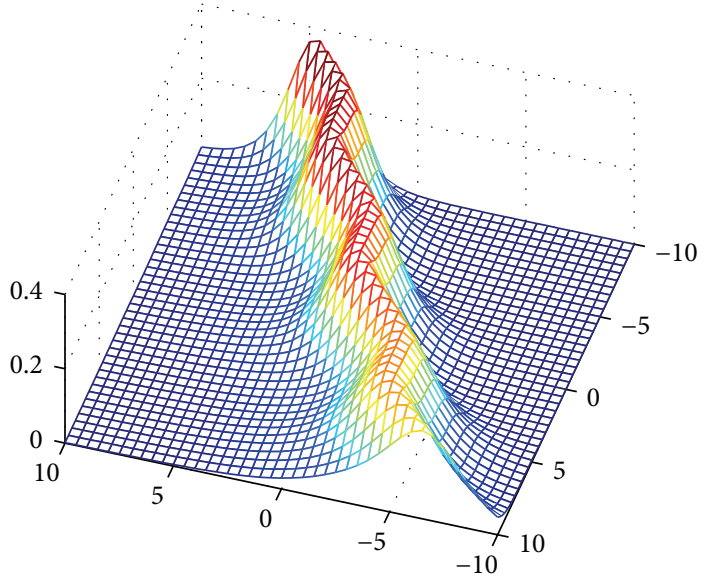

(c)

Figure 3: The process of (29) with $k_{1}=-0.8, k_{2}=0.6, k_{3}=0.4, p_{1}=p_{2}=p_{3}=0$, and $c(t)=h(t)=0$, and (a) implies $b(t)=\cos (t),(\mathrm{b})$ implies $b(t)=\cos (t)+1$, and (c) implies $b(t)=\cos (t)-1$.

From the characters of (40), we can get the following recursion formula:

$$
\bar{H}(q)= \begin{cases}\bar{H}(0) e^{\pi i n q \tau}, & q=2 n, \\ \bar{H}(1) e^{\pi i\left(2 n^{2}+2 n\right) \tau}, & q=2 n+1 .\end{cases}
$$

If we set $\bar{H}(0)=\bar{H}(1)=0$, it can satisfy with (23); that is,

$$
\begin{aligned}
\bar{H}(0) & =\sum_{n=-\infty}^{n=\infty}\left(b(t) 256 \pi^{4} n^{4} k^{4}-16 \pi^{2} n^{2} k w\right. \\
- & \left.\frac{c(t)}{30}\left(4096 \pi^{6} n^{6} k^{6}\right)+h(t) 256 \pi^{4} n^{4} k^{4} p\right) e^{2 \pi i n^{2} \tau} \\
& =0, \\
\bar{H} & (1)=\sum_{n=-\infty}^{n=\infty}\left(b(t) 16 \pi^{4}(2 n-1)^{4} k^{4}\right. \\
& -4 \pi^{2}(2 n-1)^{2} k w-\frac{c(t)}{30}\left(64 \pi^{6}(2 n-1)^{6} k^{6}\right)
\end{aligned}
$$

$$
\begin{aligned}
& \left.+h(t) 16 \pi^{4}(2 n-1)^{4} k^{4} p\right), \\
& e^{\pi i\left(2 n^{2}-2 n+1\right) \tau}=0 .
\end{aligned}
$$

With the purpose of computational convenience, set

$$
\begin{aligned}
q_{11} & =-\sum_{n=-\infty}^{n=\infty} 16 \pi^{2} n^{2} k e^{2 \pi i n^{2} \tau}, \\
q_{12} & =\sum_{n=-\infty}^{n=\infty}\left(b(t) 16 \pi^{4}(2 n-1)^{4} k^{4}\right. \\
& \left.-\frac{c(t)}{30}\left(64 \pi^{6}(2 n-1)^{6} k^{6}\right)\right) e^{\pi i\left(2 n^{2}-2 n+1\right) \tau}, \\
q_{21} & =\sum_{n=-\infty}^{n=\infty} e^{\pi i\left(2 n^{2}-2 n+1\right) \tau}, \\
q_{22} & =-\sum_{n=-\infty}^{n=\infty} 4 \pi^{2}(2 n-1)^{2} k e^{\pi i\left(2 n^{2}-2 n+1\right) \tau},
\end{aligned}
$$




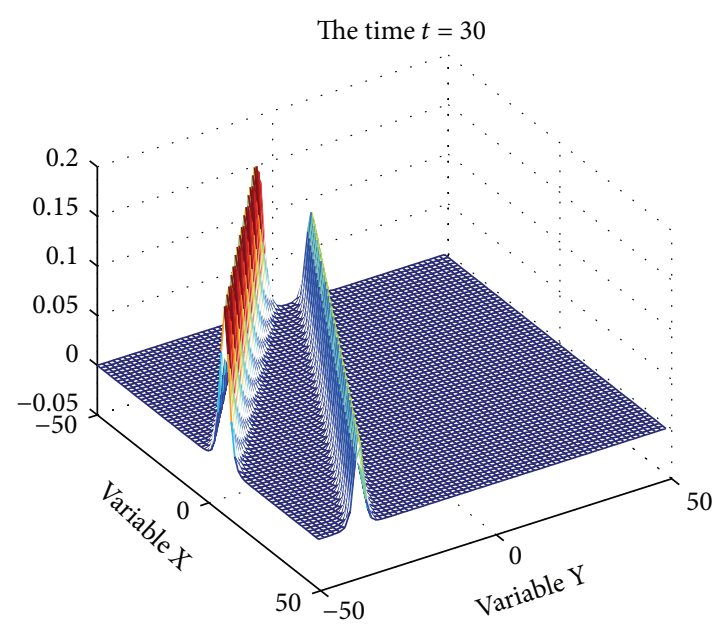

(a)

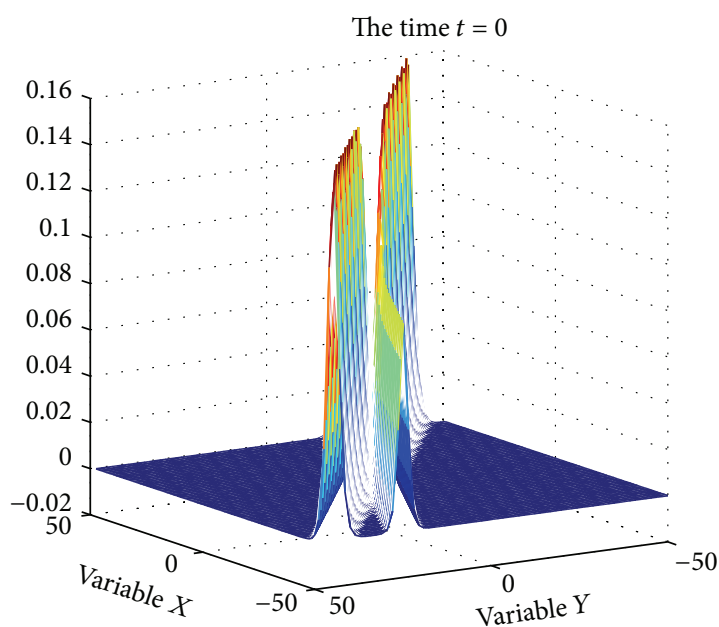

(b)

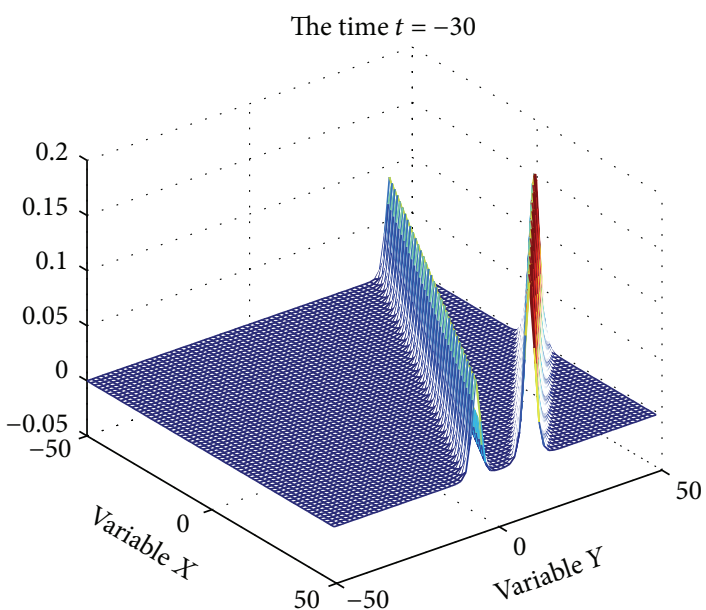

(c)

FIGURE 4: The process of (27) with $k_{1}=0.8, k_{2}=0.6, p_{1}=1.25, p_{2}=2, c(t)=30, b(t)=1$, and $h(t)=1$, and (a) implies $t=30$, (b) implies $t=0$, and (c) implies $t=-30$.

$$
\begin{aligned}
q_{31} & =\sum_{n=-\infty}^{n=\infty} e^{2 \pi i n^{2} \tau}, \\
q_{13} & =\sum_{n=-\infty}^{n=\infty}\left(b(t) 256 \pi^{4} n^{4} k^{4}-\frac{c(t)}{30}\left(4096 \pi^{6} n^{6} k^{6}\right)\right) \\
& \cdot e^{2 \pi i n^{2} \tau} .
\end{aligned}
$$

Then (42) can be changed into

$$
\begin{aligned}
q_{11} w+q_{13}+h(t) 256 \pi^{4} n^{4} k^{4} p q_{31} & =0, \\
q_{22} w+q_{12}+h(t) 16 \pi^{4}(2 n-1)^{4} k^{4} p q_{21} & =0 .
\end{aligned}
$$

The parameters $w, p$ can be got by (44) as

$$
\begin{aligned}
& w=\frac{16 n^{4} q_{12} q_{31}-(2 n-1)^{4} q_{21} q_{13}}{(2 n-1)^{4} q_{21} q_{11}-16 n^{4} q_{31} q_{22}} \\
& p=\frac{q_{12} q_{11}-q_{22} q_{13}}{h(t) \pi^{4} k^{4}\left(256 n^{4} q_{22} q_{31}-16(2 n-1)^{4} q_{11} q_{21}\right)} .
\end{aligned}
$$

Therefore, we can obtain the period wave solution of (17) in Figure 1.

\section{The Interaction of the Soliton Waves}

In this part, we will discuss the interaction of the soliton waves. From Section 3, we get the $N$-soliton solutions, which will show soliton fusion or fission when $e^{A_{i j}}=0$; on the other hand, when $e^{A_{i j}} \neq 0$, there will occur the soliton pursuing collision; after the collision, the waves are still spread along the previous direction but cannot keep the previous amplitude. Then we can describe the interaction of the soliton waves by Figures 2-5.

If $e^{A_{i j}}=0$, two-soliton solutions (27) become the following resonance solution:

$$
u=2\left[\ln \left(1+e^{\gamma_{1}}+e^{\gamma_{2}}\right)\right]_{x x} .
$$

We can analyze the soliton fusion and fission under the approximation form. 


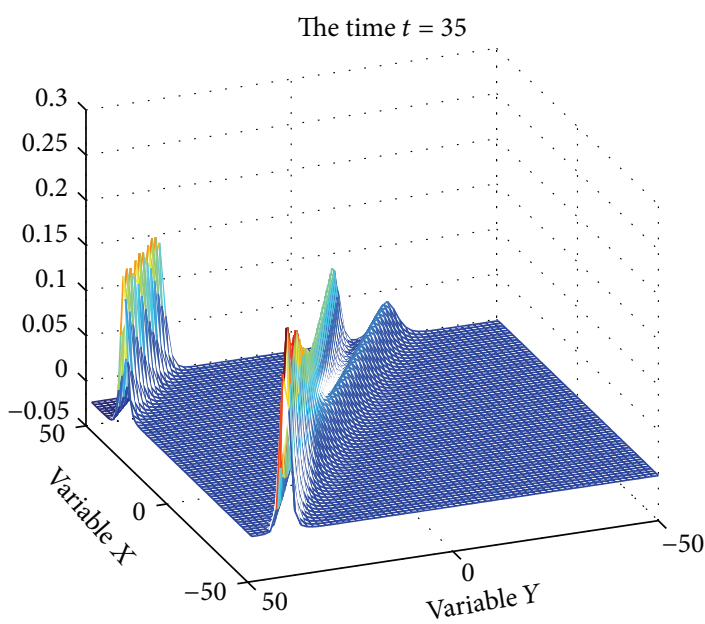

(a)

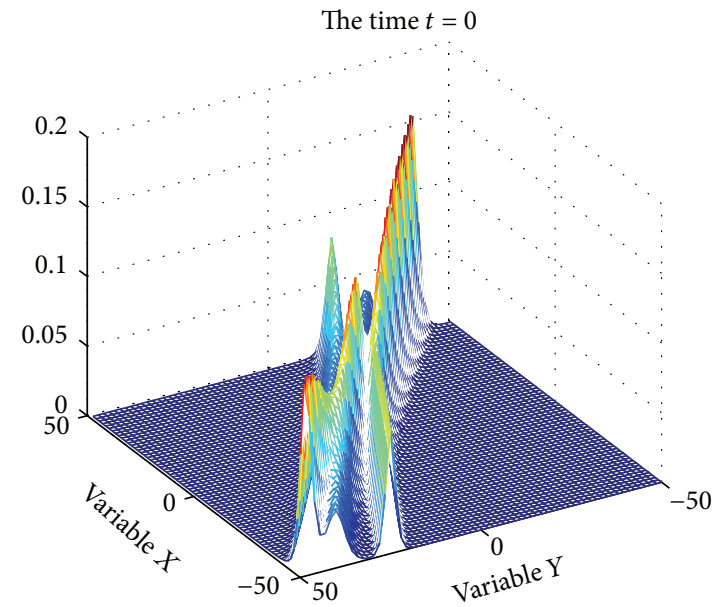

(b)

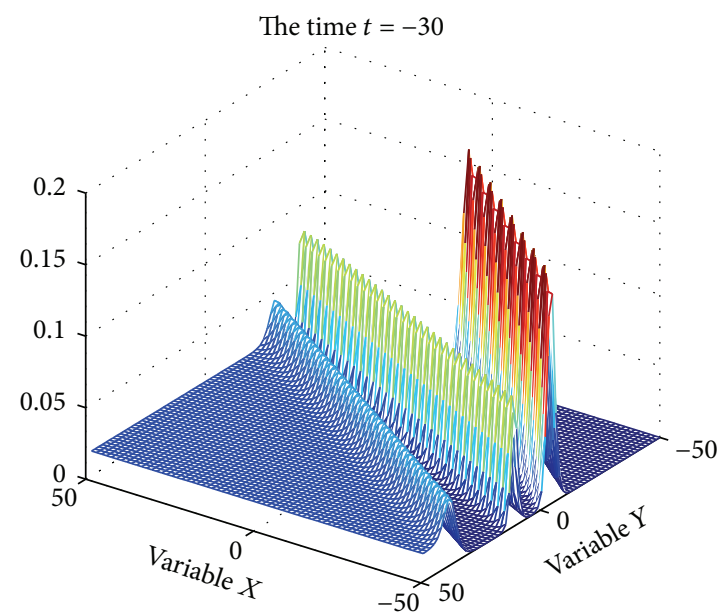

(c)

Figure 5: The process of (28) with $k_{1}=0.8, k_{2}=0.6, k_{3}=0.4, p_{1}=1.25, p_{2}=2, p_{3}=1.5, c(t)=30, b(t)=1$, and $h(t)=1$, and (a) implies $t=35$, (b) implies $t=0$, and (c) implies $t=-30$.

Figure 2(a) indicates that there appear two waves on the time 45; in Figure 2(b), it causes the collision between the two waves; during the collision, it creates new waves because of the acting force; in Figure 2(c), the two waves merge into one wave after the collision and spread placidly.

Next, we discuss the influence of the variable coefficient; due to the different coefficient, the different waves shapes will occur; the specific progress is as shown in Figure 3.

In the end, we provide the interaction of the soliton solutions based on the coefficient $e^{A_{i j}} \neq 0$. As time goes on, there will happen the soliton pursuing collision because of the different soliton speed; the wave with the faster speed will catch up with the slower speed wave; then collision of the twosoliton solution can happen; after the collision, some of the solitons can spread along the previous direction, but the other soliton will be far from the previous direction and spread with a new direction; the faster speed wave will be in front of the slower waves; Figures 4 and 5 give a visual description of the collision.
As for two-soliton solutions (27), we get the soliton pursuing collision in Figure 4.

As for three-soliton solutions (28), we describe the threesoliton pursuing collision in Figure 5.

\section{Conclusion}

In this paper, we first introduce a generalized $(2+1)$ dimensional variable-coefficient $\mathrm{KdV}$ equation, which can describe the interaction between a water wave and gravitycapillary waves better than the $(1+1)$-dimensional KdV equation. Secondly, we get the $N$-soliton solutions of the $(2+1)$-dimensional variable-coefficient $\mathrm{KdV}$ equation via the Bell-polynomial approach and explain the interactions of the $\mathrm{N}$-soliton solutions. The main conclusions of this paper can be summarized as follows:

(1) The Bell-polynomial of the $(2+1)$-dimensional variable-coefficient fifth-order $\mathrm{KdV}$ equation has 
been got based on the Bell-polynomial method; then the Bell-polynomial-typed BT is obtained by virtue of the mixing variables.

(2) $N$-soliton solutions of the $(2+1)$-dimensional variable-coefficient fifth-order $\mathrm{KdV}$ equation are obtained with the Hirota approach; then the explicit one-soliton solutions, two-soliton solutions, and three-soliton solutions are showed; under the explicit solutions and the different coefficient of $e^{A_{i j}}$, we draw the pictures of the interaction of the soliton solutions; furthermore, through the graph, we can see the soliton fusion and the fission clearly in Figure 1 with $e^{A_{i j}}=0$; in Figure 2, we analyze the influence of the variable coefficient; last but not least, the soliton pursuing collision is presented in Figures 3 and 4 and can get that, after the collision, the amplitude and the spread direction of the soliton will be changed under the condition of $e^{A_{i j}} \neq 0$.

(3) In the last part, we get the Bell-polynomial-typed BT of the variable-coefficient fifth-order $\mathrm{KdV}$ equation and via the expression of (11), we finally give rise to the bilinear-typed BT. Then, we give the period wave solutions with the help of the Riemann theta function.

\section{Conflict of Interests}

The authors declare that there is no conflict of interests regarding the publication of this paper.

\section{Acknowledgments}

This work was supported by the National Natural Science Foundation of China (no. 11271007), Special Funds for Theoretical Physics of the National Natural Science Foundation of China (no. 11447205), SDUST Research Fund (no. 2012KYTD105), and Graduate Innovation Foundation from Shandong University of Science and Technology (no. YC140327).

\section{References}

[1] M. J. Ablowitz and P. A. Clarkson, Solitons, Nonlinear Evolution Equations and Inverse Scattering, Cambridge University Press, New York, NY, USA, 1991.

[2] C. Rogers and W. F. Shadwick, Backlund Transformations and Their Applications, Academic Press, London, UK, 1982.

[3] R. M. Miura, Bäcklund Transformation, Springer, Berlin, Germany, 1978.

[4] A. Choudhuri and K. Porsezian, "Dark-in-the-bright solitary wave solution of higher-order nonlinear Schrödinger equation with non-Kerr terms," Optics Communications, vol. 285, no. 3, pp. 364-367, 2012.

[5] Z. Li, L. Li, H. Tian, and G. Zhou, "New types of solitary wave solutions for the higher order nonlinear Schrödinger equation," Physical Review Letters, vol. 84, no. 18, pp. 4096-4099, 2000.

[6] Z. H. Xu, B. S. Yin, and Y. J. Hou, "Highly nonlinear internal solitary waves over the continental shelf of the northwestern
South China Sea," Chinese Journal of Oceanology and Limnology, vol. 28, no. 5, pp. 1049-1054, 2010.

[7] H. W. Yang, D. Z. Yang, Y. L. Shi, S. S. Jin, and B. S. Yin, "Interaction of algebraic Rossby solitary waves with topography and atmospheric blocking," Dynamics of Atmospheres and Oceans, vol. 71, pp. 21-34, 2015.

[8] R. Hirota, "Exact solution of the korteweg-de vries equation for multiple collisions of solitons," Physical Review Letters, vol. 27, no. 18, pp. 1192-1194, 1971.

[9] R. Hirota, "Exact $N$-soliton solutions of the wave equation of long waves in shallow-water and in nonlinear lattices," Journal of Mathematical Physics, vol. 14, pp. 810-814, 1973.

[10] W.-X. Ma and Y. C. You, "Solving the Kortewegõde Vries equation by its bilinear form: wronskian solutions," Transactions of the American Mathematical Society, vol. 357, no. 5, pp. 17531778, 2005.

[11] H.-H. Dong and X. Z. Wang, "Lie algebra and Lie super algebra for the integrable couplings of NLS-MKDV Hierarchy," Communications in Nonlinear Science and Numerical Simulation, vol. 14, no. 12, pp. 4071-4077, 2009.

[12] H. H. Dong, Y. F. Zhang, Y. F. Zhang, and B. S. Yin, "Generalized bilinear differential operators, binary bell polynomials, and exact periodic wave solution of boiti-leon-manna-pempinelli equation," Abstract and Applied Analysis, vol. 2014, Article ID 738609, 6 pages, 2014

[13] H. H. Dong and Y. F. Zhang, "Exact periodic wave solution of extended $(2+1)$-dimensional shallow water wave equation with generalized $D_{p}$ operator," Communications in Theoretical Physics, vol. 63, pp. 401-405, 2015.

[14] H. Q. Zhaoa, "Soliton propagation and collision in a variablecoefficient coupled Korteweg-de Vries equation," The European Physical Journal B, vol. 85, pp. 302-308, 2012.

[15] W. X. Ma, "Generalized bilinear differential equations," Studies in Nonlinear Sciences, vol. 2, no. 4, pp. 140-144, 2011.

[16] W.-X. Ma, "Bilinear equations, bell polynomials and linear superposition principle," Journal of Physics: Conference Series, vol. 411, no. 1, pp. 473-476, 2013.

[17] E. G. Fan, "The integrability of nonisospectral and variablecoefficient KdV equation with binary Bell polynomials," Physics Letters A, vol. 375, no. 3, pp. 493-497, 2011.

[18] E. G. Fan and Y. C. Hon, "Super extension of Bell polynomials with applications to supersymmetric equations," Journal of Mathematical Physics, vol. 53, no. 1, Article ID 013503, 2012.

[19] Y. H. Wang and Y. Chen, "Integrability of the modified generalised Vakhnenko equation," Journal of Mathematical Physics, vol. 53, no. 12, Article ID 123504, 2012.

[20] Y.-H. Wang and Y. Chen, "Binary Bell polynomials, bilinear approach to exact periodic wave solutions of $(2+1)$-dimensional nonlinear evolution equations," Communications in Theoretical Physics, vol. 56, no. 4, pp. 672-678, 2011.

[21] Y.-H. Wang and Y. Chen, "Bäcklund transformations and solutions of a generalized Kadomtsev-Petviashvili equation," Communications in Theoretical Physics, vol. 57, no. 2, pp. 217222, 2012.

[22] E. Fan, "New bilinear Bäcklundtrans transformation and Lax pair for the supersymmetric two-BOSon equation," Studies in Applied Mathematics, vol. 127, no. 3, pp. 284-301, 2011.

[23] Y. L. Song and M. A. Han, "Periodic solutions of periodic delay predator-prey system with nonmonotonic functional response," Journal of Shanghai Jiaotong University, vol. E-8, pp. 107-110, 2003. 
[24] H. Wang and T. C. Xia, "Bell polynomial approach to an extended Kortewegõde Vries equation," Mathematical Methods in the Applied Sciences, vol. 37, no. 10, pp. 1476-1487, 2014.

[25] Y. H. Wang and Y. Chen, "Binary Bell polynomial manipulations on the integrability of a generalized $(2+1)$-dimensional Korteweg-de Vries equation," Journal of Mathematical Analysis and Applications, vol. 400, no. 2, pp. 624-634, 2013.

[26] H. W. Yang, X. R. Wang, and B. S. Yin, "A kind of new algebraic Rossby solitary waves generated by periodic external source," Nonlinear Dynamics, vol. 76, no. 3, pp. 1725-1735, 2014.

[27] W.-X. Ma, "Nonlinear continuous integrable Hamiltonian couplings," Applied Mathematics and Computation, vol. 217, no. 17, pp. 7238-7244, 2011.

[28] X. R. Wang, Y. Fang, and H. H. Dong, "Component-trace identity for Hamiltonian structure of the integrable couplings of the Giachetti-Johnson (GJ) hierarchy and coupling integrable couplings," Communications in Nonlinear Science and Numerical Simulation, vol. 16, no. 7, pp. 2680-2688, 2011.

[29] X. R. Wang, X. E. Zhang, and P. Y. Zhao, "Binary nonlinearization for AKNS-KN coupling system," Abstract and Applied Analysis, vol. 2014, Article ID 253102, 12 pages, 2014.

[30] H. W. Yang, B. S. Yin, and Y. L. Shi, "Forced dissipative Boussinesq equation for solitary waves excited by unstable topography," Nonlinear Dynamics, vol. 70, no. 2, pp. 1389-1396, 2012.

[31] A. Nakamura, "A direct method of calculating periodic wave solutions to nonlinear evolution equations. I. Exact twoperiodic wave solution," Journal of the Physical Society of Japan, vol. 47, no. 5, pp. 1701-1705, 1979. 


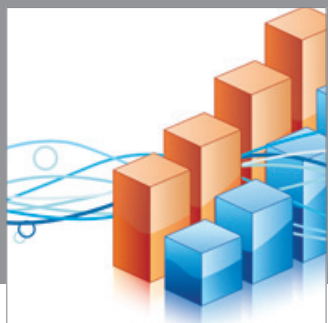

Advances in

Operations Research

mansans

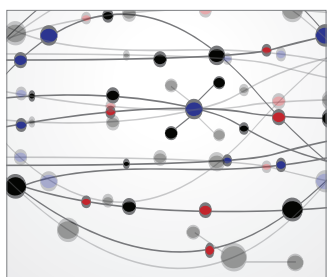

The Scientific World Journal
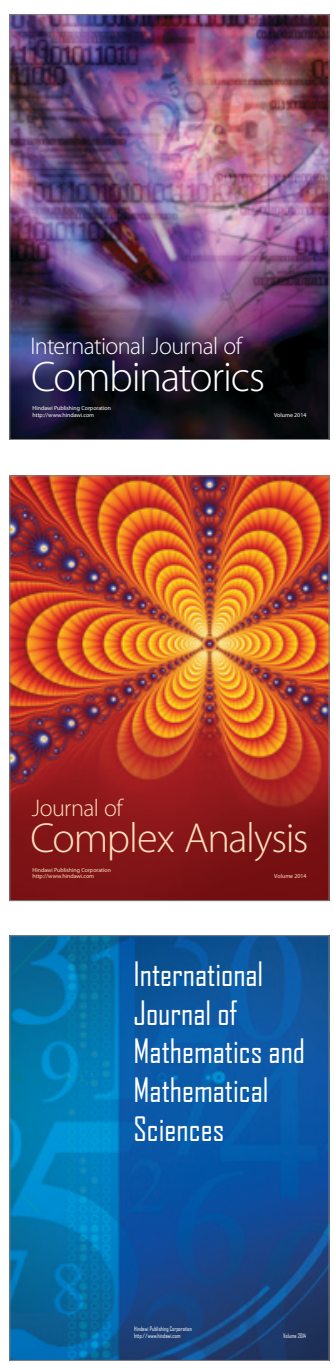
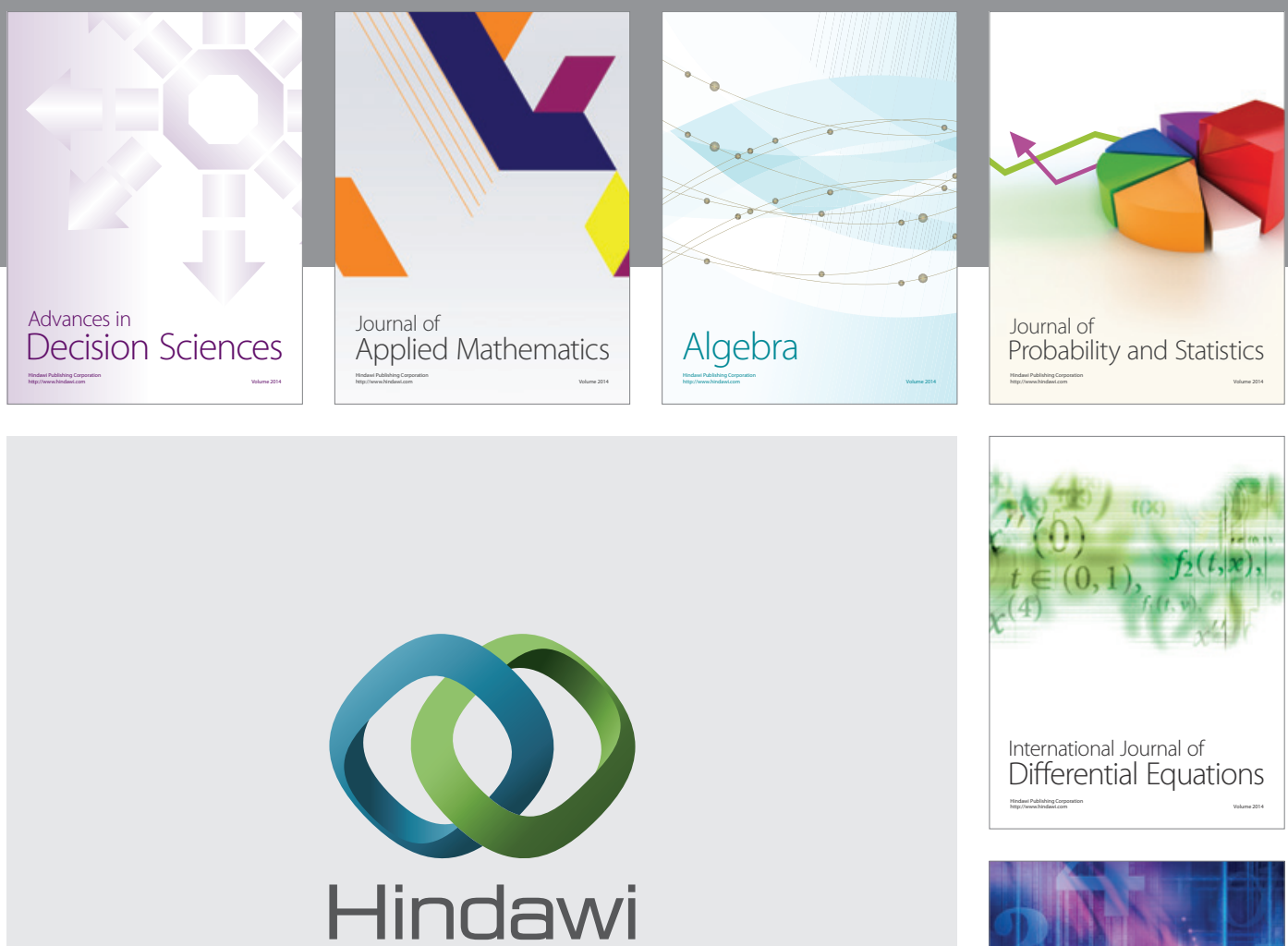

Submit your manuscripts at http://www.hindawi.com
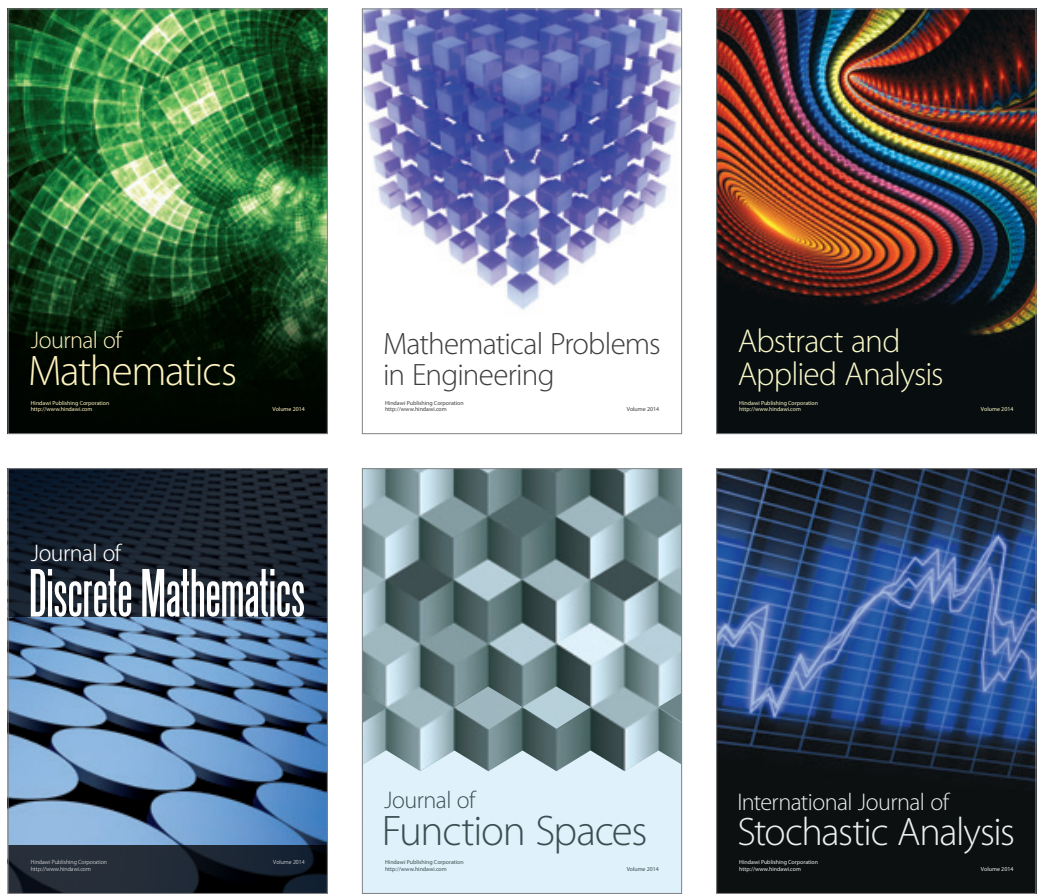

Journal of

Function Spaces

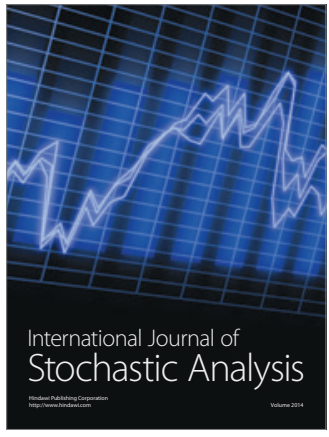

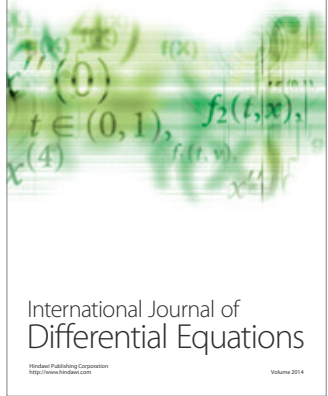
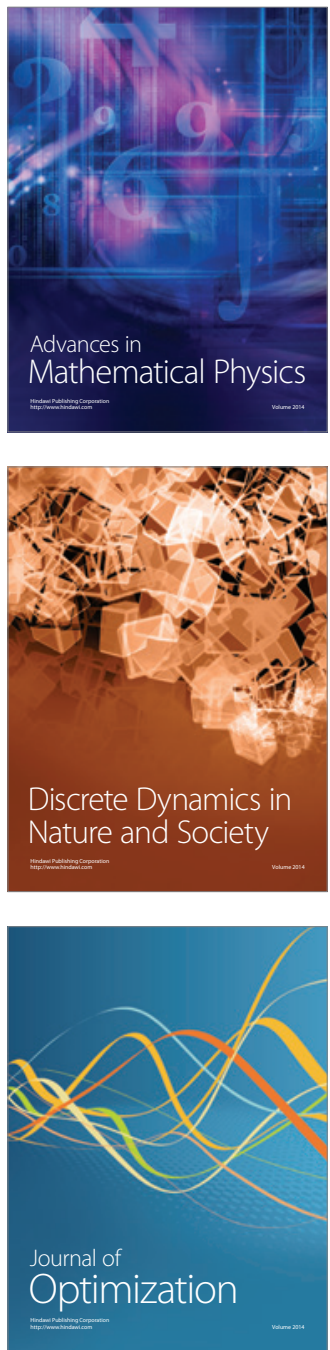